\title{
Comparaison de trois types de pièges pour l'échantillonnage des populations de pucerons ailés
}

\author{
G. Labonne 1, F. Lauriaut ${ }^{1}$ et J. Quiot 2 \\ 1 INRA, Laboratoire de recherches de la chaire d'écologie animale et de zoologie agricole, ENSAM, 34060 Montpellier Cedex; \\ 2 INRA, Laboratoire de virologie, LPRC, CIRAD, BP 5035, 34032 Montpellier, France
}

(reçu le 15 décembre 1987, accepté le 31 mars 1989)

\begin{abstract}
Résumé - Trois modèles de pièges ont été essayés pour échantillonner les populations de pucerons ailés près de la végétation : piège à succion, piège jaune à eau, piège à fils englués. L'essai a été réalisé en juin dans le sud-est de la France. L'analyse de la répartition des captures a été faite sur 55000 pucerons, classés en 93 espèces ou groupes d'espèces. Deux critères ont été utilisés : l'analyse factorielle des correspondances appliquée aux échantillons des 3 types de pièges, et l'étude des corrélations entre les échantillons provenant du piège jaune ou des pièges à fils englués relativement à ceux du piège à succion. Ces 2 critères indiquent que l'échantillon obtenu par les pièges à fils englués diffère peu de celui obtenu par le piège à succion. La répartition des espèces dans le piège jaune est, en revanche, différente. Elle se caractérise par l'abondance de quelques espèces (particulièrement Aphis citricola Van der Goot), tandis que d'autres sont presque absentes. L'analyse des variations quotidiennes des espèces les plus abondantes montre que plus le nombre d'individus capturés est grand, meilleures sont les corrélations entre les différents pièges, sans distinction entre pièges attractifs et pièges neutres. Ces résultats montrent que les pièges à fils englués constituent une technique adéquate de substitution aux pièges à succion pour étudier l'ensemble de l'aphidofaune près de la végétation.
\end{abstract}

aphides - piégeage - piège à succion - piège jaune - piège à fils englués

Summary - Comparison of three types of traps to sample alate aphid populations. Three types of traps have been used to sample alate aphid populations flying near plant canopy: suction trap, yellow water pan, sticky threads trap. These traps were operated during 21 days in June at Montpellier, in southeastern France. The 55000 aphids which were caught were identified and distributed in 93 species or groups of species. Their distribution in the 3 types of traps has been compared using 2 methods: the factorial analysis and the study of correlation between the samples of the different types of traps. These 2 methods give evidence that the samples obtained by the sticky threads traps and by the suction trap are very similar. The distribution of aphids in the yellow water pan is more different, and is characterized by a few number of abundant species (particularly Aphis citricola Van der Goot) and other species almost missing. The analysis of daily variations for the most abundant species shows that the higher is the number of aphids, the better are the correlations between the samples from the traps, without distinction between attractive or impaction traps. From these results, it appears that the sticky threads traps may be used as a substitute of the suction traps to study the aphid populations flying near the vegetation canopy.

aphid - suction trap - yellow water trap - stickey fishing line traps

\section{Introduction}

Les virus transmis sur le mode non persistant sont disséminés de plante à plante dans les parcelles cultivées par leurs vecteurs, les pucerons. L'étude de la dissémination de ces virus nécessite donc le recueil de données sur les fluctuations des vols de pucerons près des plantes.
Par ailleurs, les essais en conditions contrôlées ont montré que ces virus peuvent être transmis par plusieurs espèces de pucerons. Les études réalisées en conditions naturelles ont confirmé que de nombreuses espèces ont un rôle de vecteur (Adlerz, 1986; Halbert et al., 1981; Harrington et al., 1986; Knoke et al., 1977; Labonne et al., 1982a; Raccah et al., 1985). Les 
techniques de piégeage des vecteurs mises en œuvre doivent donc rendre compte des variations de populations pour l'ensemble des espèces présentes. Or, il existe une gamme étendue de types de pièges, dont les principes de fonctionnement diffèrent (Taylor et Palmer, 1972; Robert et al., 1988).

L'objet de cet article est de présenter une comparaison de 3 types de pièges pour l'évaluation des populations de pucerons ailés présentes près de la végétation :

- piège à succion : son principe, qui est de capter et filtrer un volume d'air pris au-dessus de lui avec une force suffisante pour que les insectes ne s'échappent pas, en fait une référence pour l'échantillonnage de l'air à vitesse de vent constante;

- piège jaune à eau : ce type de piège est celui qui a été le plus fréquemment associé aux études sur la dissémination des virus;

- piège à fils englués : piège d'interception que nous avons choisi pour ses qualités de porosités optique et mécanique.

La répartition par espèce des échantillons recueillis par les 3 types de pièges et les variations d'effectifs des principales espèces sont successivement analysées.

\section{Matériel et Méthodes}

Trois modèles de pièges ont été comparés (Fig. 1) : - un piège à succion précédemment décrit (Labonne et al., 1982b). Son orifice d'aspiration de $30 \mathrm{~cm}$ de diamètre est situé à $25 \mathrm{~cm}$ du sol et son débit est de $1080 \mathrm{~m}^{3 / \mathrm{h}}$ par vent nul;

- des pièges jaunes à eau : bacs carrés de $60 \mathrm{~cm}$ de côté et $10 \mathrm{~cm}$ de haut, gris à l'extérieur, jaune uboutond'or» à l'intérieur (teinte caractérisée par Brunel et Langouet, 1970). Ils sont remplis d'eau déminéralisée sans mouillant;

- des pièges à fils englués précédemment décrits (Labonne et al., 1983). Ils sont accrochés à des supports verticaux, mettant leur surface piégeante (25 $\times 25 \mathrm{~cm}$ ) entre le sol et $160 \mathrm{~cm}$ de hauteur. L'engluage est effectué à l'aide d'une glu sous pression (Tangle-trap). Le solvant utilisé pour décoller les insectes est l'essence de térébenthine.

Le terrain d'essai, situé à Montpellier (sud-est de la France), est constitué d'une pelouse composite (incluant d'autres espèces que des graminées) maintenue à $10 \mathrm{~cm}$ de haut. Outre les pièges étudiés, il comporte un piège à succion de 12,2 $\mathrm{m}$ de haut (de type "Rothamsted Insect Survey") et une station fournissant les données météorologiques. II présente les caractéristiques de dégagement nécessaires au fonctionnement de ces 2 systèmes de recueil de données.

Les pièges à fils englués sont espacés de $3 \mathrm{~m}$ et placés suivant 2 directions orthogonales $(1,2,3,7,8$, $9, A, C, F$ sont orientés est-ouest; $4,5,6, B, D, E$ sont orientés nord-sud) pour tenir compte des variations de direction du vent et éventuellement de différences liées
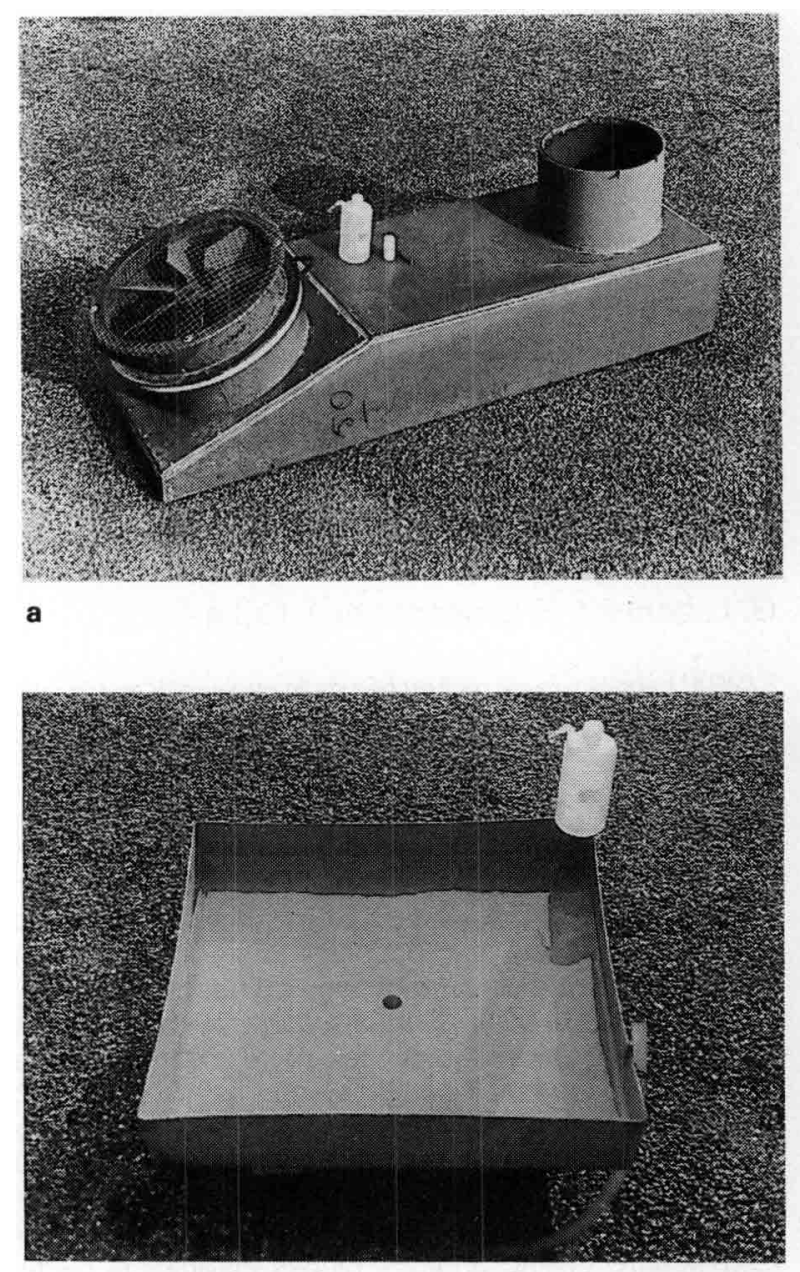

b

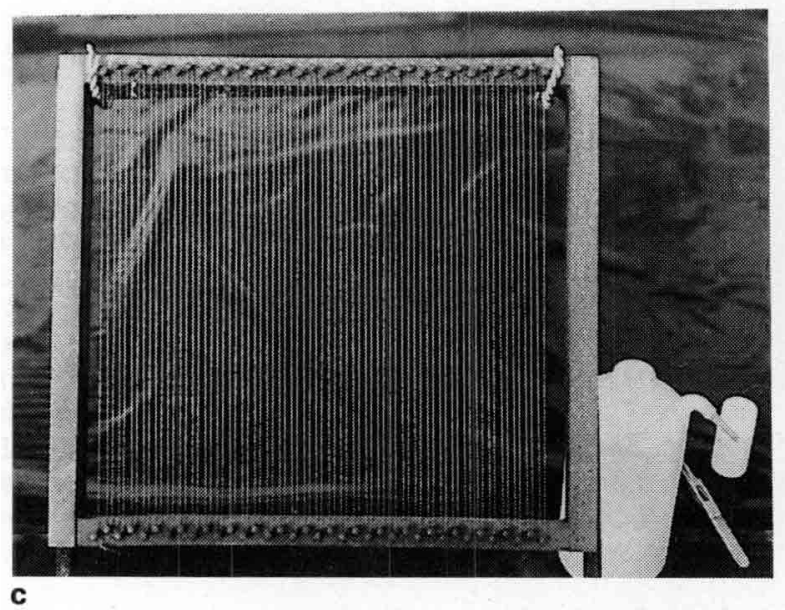

Fig. 1. Les 3 modèles de pièges utilisés : a : piège à succion; $\mathrm{b}$ : piège jaune à eau; $\mathrm{c}$ : piège à fils englués.

à la réflexion de la lumière en fonction de la position du soleil. Leur surface piégeante est placée à différentes hauteurs, de façon à encadrer la strate échantillonnée par le piège à succion :

-0 à $25 \mathrm{~cm}$ pour les pièges $1,4,7$;

-30 à $55 \mathrm{~cm}$ pour les pièges $A, B, C, D, E, F$;

-70 à $95 \mathrm{~cm}$ pour les pièges $2,5,8$;

-135 à $160 \mathrm{~cm}$ pour les pièges $3,6,9$. 
Le nombre des pièges de la strate $30-55 \mathrm{~cm}$, a priori la plus comparable à celle échantillonnée par le piège à succion, a été porté à 6 de façon à travailler sur des effectifs suffisants.

Le piège à succion est à $3 \mathrm{~m}$ des pièges les plus proches (A et $C$ ).

Les 2 pièges jaunes à eau sont à $6 \mathrm{~m}$ des pièges les plus proches. L'un est posé sur le sol, l'autre est à $70 \mathrm{~cm}$ au-dessus du sol.

Les piégeages ont été réalisés pendant 21 journées entre le 6 et le 29 juin 1984, période d'abondance et de diversité maximales des aphides dans le sud-est de la France. Les pièges étaient nettoyés ou remplacés le matin à $10 \mathrm{~h}$, heure locale.

Les pucerons capturés ont été conservés dans l'alcool à $70^{\circ}$ et leur identification a été réalisée à la loupe binoculaire.

\section{Résultats}

Les 55000 pucerons capturés durant les 21 journées de prélèvements ont été identifiés et répartis en 93 espèces ou groupes d'espèces (Tableau I). Au cours de cette période, la vitesse moyenne du vent a été de $1,6 \mathrm{~m} / \mathrm{sec}$ à $2 \mathrm{~m}$ de haut.

\section{Influence de la position des pièges à fils en- glués et des pièges jaunes à eau sur l'échan- tillonnage}

L'analyse de la répartition des espèces dans les échantillons des 15 pièges à fils englués conduit aux conclusions suivantes.

Des hétérogénéités de répartition des espèces existent entre les pièges de même orientation situés à la même hauteur $\left(\chi^{2}\right.$ significatifs à 0,05$)$ (Tableau II). Ces hétérogénéités non imputables à un facteur contrôlé sont cependant modérées, ainsi que le montrent aussi les corrélations entre les échantillons de ces pièges, qui varient entre 0,87 et 0,93 (calcul sur les effectifs des 93 espèces, transformés en Log $(n+1))$.

Tableau II. Répartitions comparées des espèces dans les pièges à fils englués de même orientation et de même hauteur pris 2 à 2 . Test du $\chi^{2}$ sur les espèces, dont l'effectif est $\geq 4$ dans chaque échantillon.

\begin{tabular}{lrcc}
\hline Pièges & $\chi^{2}$ & $D D L$ & $\begin{array}{c}\text { Valeur limite } \\
\text { à } 0,05\end{array}$ \\
\hline PF1 / PF7 & 41,8 & 28 & 41,3 \\
PFA / PFC & 72,0 & 29 & 42,6 \\
PFA / PFF & 81,0 & 31 & 45,0 \\
PFC / PFF & 134,5 & 32 & 46,2 \\
PFB / PFD & 31,4 & 23 & 35,2 \\
PFB / PFE & 35,4 & 25 & 37,7 \\
PFD / PFE & 23,1 & 24 & 36,4 \\
PF2 / PF8 & 90,7 & 43 & 62,5 \\
PF3 / PF9 & 67,3 & 41 & 60,1 \\
\hline
\end{tabular}

L'orientation des pièges affecte peu la répartition des espèces : une analyse factorielle des correspondances appliquée aux 15 échantillons ne permet pas de mettre en évidence un axe correspondant à ce facteur, et le coefficient de corrélation entre les 2 échantillons obtenus par le regroupement des pièges suivant leur orientation est de 0,96 (calcul sur les effectifs des 93 espèces, transformés en $\log (n+1))$.

La répartition des espèces est davantage modifiée par l'altitude des pièges : l'altitude correspond à l'axe 1 de l'analyse factorielle des correspondances précédemment citée $(51 \%$ de l'inertie du nuage de points). Des gradients altitudinaux différents suivant les espèces existent donc. Les coefficients de corrélation entre les échantillons obtenus par le regroupement des pièges de même hauteur indiquent que les différences sont elles aussi modérées.

L'existence de gradients altitudinaux implique que les strates d'air échantillonnées par les pièges soient les mêmes pour que ceux-ci puissent être comparés, ou que les différences observées soient interprétées relativement aux gradients.

Le choix de la strate $30-55 \mathrm{~cm}$ pour la comparaison entre les pièges à fils englués et le piège à succion est justifié à la fois par un argument logique (base de la zone d'aspiration du piège à succion à $25 \mathrm{~cm}$ ) et par la comparaison des échantillons capturés par les pièges à fils englués des différentes strates et l'échantillon du piège à succion (Tableau III).

Les coefficients de corrélation entre les 2 échantillons des pièges jaunes au sol et à $70 \mathrm{~cm}$ et celui du piège à succion sont respectivement de 0,704 et 0,709 . Ils ne permettent pas d'évaluer quelle est la strate la plus comparable à celle échantillonnée par le piège à succion. Compte tenu du fait que les pièges jaunes à eau capturent les pucerons qui volent au-dessus d'eux, il est cependant plus logique de comparer le piège jaune posé au sol au piège à succion et

Tableau III. Coefficients de corrélation entre les effectifs transformés en Log $(n+1)$ des 93 espèces obtenues par le piège à succion et par les pièges à fils englués placés à différentes hauteurs.

\begin{tabular}{lcccc}
$\begin{array}{l}\text { Piège } \\
\text { Hauteur en cm }\end{array}$ & $\begin{array}{c}P F \\
0\end{array}$ & $\begin{array}{c}P F \\
30\end{array}$ & $\begin{array}{c}P F \\
70\end{array}$ & $\begin{array}{c}P F \\
135\end{array}$ \\
\hline PF 0 & 1,00 & 0,93 & 0,88 & 0,83 \\
PS & 0,86 & 0,91 & 0,85 & 0,83
\end{tabular}

PF : piège à fils englués; PS : piège à succion. 
Tableau l. Comparaison des captures de pucerons ailés réalisées par des pièges placés sur une pelouse composite de $10 \mathrm{~cm}$ de haut, en juin 1984 (21 jours de prélèvement) à Montpellier.

\begin{tabular}{|c|c|c|c|}
\hline $\begin{array}{l}\text { PIEUES } \\
\text { ALTITUDE, } \\
\text { ORIENTATION } \\
\end{array}$ & $\begin{array}{l}\text { PF: } \\
\text { SOL } \\
\mathrm{Z}-4\end{array}$ & $\begin{array}{l}P F> \\
S O L \\
Q-W\end{array}$ & $\begin{array}{l}\text { PFA } \\
\text { SOL } \\
\mathrm{N}-\mathrm{S} \\
\end{array}$ \\
\hline Acyethoslphon ouphorblan & 1 & o & $\circ$ \\
\hline Acyrthosiphon plsum & 4 & 7 & 2 \\
\hline Acyetnosiphon iactucas & 0 & 0 & o \\
\hline Acyrenosiphon sp. & 4 & 2 & 1 \\
\hline $\begin{array}{l}\text { ADELGIDAE } \\
\text { Amphorophor rub! }\end{array}$ & 3 & 1 & 4 \\
\hline Amphorophore rub! & 0 & 0 & 0 \\
\hline Anoocla sp. & 13 & 14 & 9 \\
\hline Apnis clericola & 53 & 102 & 56 \\
\hline Apnia cractas & 1 & 2 & 2 \\
\hline Aphis crocclvora (groupa) & 23 & 26 & 33 \\
\hline Aphls cye 1 sorum & 25 & 24 & 10 \\
\hline Aphis rabae (groupa) & 38 & 38 & 28 \\
\hline Apnis gossypti (groupe) & 48 & 41 & 42 \\
\hline Aphis noril & 2 & 2 & 2 \\
\hline Aphls pom 1 & 0 & 2 & 1 \\
\hline Aphis ruborum (?) & 5 & 9 & 2 \\
\hline Aphf a ambuci & o & 0 & o \\
\hline Aphis verbascl & o & 1 & 1 \\
\hline Aphis rumicis & 6 & 5 & $\mathbf{8}$ \\
\hline Apni ap. & 47 & 57 & 50 \\
\hline Aphlo (Protaphis) ap. & 3 & 3 & 4 \\
\hline Aploneura lentsscl & 10 & 24 & 24 \\
\hline Appendisete robiniag & 28 & 40 & 36 \\
\hline Acheroldes ap. & 1 & 0 & 0 \\
\hline Aulacorehum solani & 0 & 0 & 0 \\
\hline Brachycaudus anygdalínus & 15 & 9 & e \\
\hline Brachycaudus cirdul & 13 & 15 & 3 \\
\hline Brachycaudus nellchryal & 17 & 24 & 3 \\
\hline Brachycaudus op. & 0 & 2 & 1 \\
\hline Brachyungula tamaricio & 1 & 0 & $\circ$ \\
\hline Brevicoryno bressicae & 2 & 1 & 1 \\
\hline Capicopnorus oloegn! & 0 & $\circ$ & 4 \\
\hline Cavarialia agopodil & 2 & 1 & 4 \\
\hline Cavariella incermedia & 0 & $\circ$ & 0 \\
\hline Cavarialia theobaldi & o & 0 & 0 \\
\hline Chalrophorus popull-albae & 0 & 0 & o \\
\hline Cnalropnorus ep. & 2 & 1 & 0 \\
\hline Clnare sp. & 3 & 0 & 5 \\
\hline Colorados sp. & o & 0 & 0 \\
\hline Dluraphla noxla & 40 & 22 & 31 \\
\hline Dysaphls sp. & 17 & 17 & 11 \\
\hline EF $1000 \mathrm{ma} \mathrm{sp}$ & 0 & 1 & o \\
\hline Euca11iptarus t111.a & 4 & $\circ$ & $\circ$ \\
\hline Everazzia olegana & 0 & 0 & o \\
\hline Eulachnus ap. & 4 & 4 & 5 \\
\hline Hayhuret: etrlplicta & o & 0 & $\circ$ \\
\hline Hoplocallis plcqua & 0 & 1 & 1 \\
\hline Hyodepots sp. & 24 & 43 & 23 \\
\hline Hystopterus prunl & 4 & 5 & a \\
\hline Myperomyzus lactucas & 5 & 3 & 4 \\
\hline Hyperonyzus pleridis & 2 & 5 & 4 \\
\hline izYponia sp. & 3 & 2 & 1 \\
\hline L10somapnla berberdd1a & 0 & o & 1 \\
\hline LIPEphIs arysimf & $\circ$ & $\circ$ & $\circ$ \\
\hline $\begin{array}{l}\text { Macrosiphum eupnorbleo } \\
\text { macrosipnum roseo }\end{array}$ & 0 & 1 & 1 \\
\hline hacrosipnum rosao & 1 & 0 & 1 \\
\hline Mecrosiphonlalla tapuskae & 0 & 0 & 0 \\
\hline Megoura viclae & 0 & 0 & 0 \\
\hline Melanaphis donacts & 1 & 3 & 3 \\
\hline Metopolophlum dirnodum & 2 & 2 & $\circ$ \\
\hline macopolophium festucan & 3 & 3 & 3 \\
\hline Myzocallis castanteola & 2 & 2 & 3 \\
\hline nyzocsl11s sp. & 0 & 0 & 0 \\
\hline nyzue corasi 1 & 1 & 1 & 0 \\
\hline Myzus byene! & 9 & 1 & 0 \\
\hline Myzus parsicae & 0 & 1 & 2 \\
\hline Nasonovia Fiolg-nigrt & 0 & $\circ$ & 3 \\
\hline Nearciephis bakerl & 1 & 2 & 1 \\
\hline Ovarus cratagarius & 0 & $\circ$ & o \\
\hline PEMPMIGINAE & 10 & 3 & 5 \\
\hline Pharodan humull & 0 & 1 & o \\
\hline PHYLLOXERIDAE & 11 & 5 & $\circ$ \\
\hline Pleot 1 chophorus glandulasus & 0 & 1 & 0 \\
\hline Rhopalomyzus poas & 0 & 0 & 1 \\
\hline Rnopelosipnum insertum & 0 & 0 & 0 \\
\hline Fhoplosiphum padl & 20 & 35 & e \\
\hline Schizaphla gramlnum & 0 & 2 & 1 \\
\hline Semiapnis dauel & 8 & 7 & a \\
\hline S1pha $\log$ ang & 10 & 7 & ○ \\
\hline s!pre naldis & i & 0 & 1 \\
\hline Sitoolon avenes & 3 & 1 & 2 \\
\hline Staggerlella necopinate & 0 & 0 & 0 \\
\hline Taxacal11a sp. & 2 & 2 & 0 \\
\hline Tecranour* ap. & 3 & 5 & 4 \\
\hline Thelexes sp. & 2 & 0 & 1 \\
\hline Therloephle trifolld & 18 & 21 & 22 \\
\hline Thorioepnia ep. & 1 & 2 & 0 \\
\hline Trams ep. & 0 & 0 & 0 \\
\hline Tuboreulatus egblerl & 0 & 0 & 0 \\
\hline Uroleucon ap. & 1 & 1 & 1 \\
\hline Uronelan sp. & 2 & 5 & 2 \\
\hline Menlgrentelita ep. & 0 & 0 & 0 \\
\hline AUTRES & 2 & 2 & 1 \\
\hline TOTAL & 596 & $\Delta 82$ & 20 \\
\hline
\end{tabular}


aux pièges à fils englués situés entre 30 et 55 $\mathrm{cm}$ : le piège jaune à $70 \mathrm{~cm}$ exclut de son échantillonnage les strates inférieures.

\section{Comparaison de la répartition des espèces dans les pièges}

Comparaison par l'analyse factorielle des correspondances de la répartition par espèces des pucerons capturés par les différents pièges

Le piège à succion, les pièges à fils englués placés entre 30 et $55 \mathrm{~cm}$ et le piège jaune à eau situé sur le sol ont été placés en variables actives. Le piège jaune à eau à $70 \mathrm{~cm}$ est mis en variable supplémentaire, pour le situer par rapport aux autres.

Analyse de l'ensemble des espèces. Une première analyse effectuée avec toutes les espèces fait ressortir le cas particulier de Aphis citricola Van der Goot. Cette espèce est très fortement capturée par le piège jaune au sol (points confondus sur la représentation graphique de l'analyse), et sa masse est responsable d'une grande partie de l'inertie du système (Tableau IV). Le piège jaune à $70 \mathrm{~cm}$ est représenté au même endroit.

Analyse après élimination de A. citricola. La représentation des 2 premiers axes de l'analyse factorielle pour les autres espèces (Fig. 2) montre que les 3 types de pièges se distinguent les uns des autres.

L'axe 1 (abscisses) correspond à l'opposition entre les espèces plus abondantes dans le piège jaune au sol que dans les autres; l'axe 2 (ordonnées), à l'opposition entre celles plus abondantes dans les pièges à fils que dans le piège à succion. Les pourcentages d'inertie portés par ces 2 axes (Tableau IV) montrent que l'échantillonnage des pièges à fils englués est plus proche de celui du piège à succion que ne l'est celui du piège jaune au sol. Le piège jaune à $70 \mathrm{~cm}$ est près du piège jaune au sol. Les 6 pièges à fils englués

Tableau IV. Comparaison des effectifs des 93 espèces de pucerons capturées par les 6 pièges à fils englués à $30 \mathrm{~cm}$, par le piège jaune au sol et par le piège à succion : pourcentages d'inertie portés par les 3 axes principaux de l'analyse factorielle des correspondances.

Axe

Pourcentage d'inertie porté par l'axe

\begin{tabular}{rrr} 
& \multicolumn{2}{c}{ Aphis citricola } \\
& Inclus & Exclu \\
\hline 1 & 84,00 & 66,10 \\
2 & 11,19 & 23,96 \\
3 & 1,54 & 3,14 \\
\hline
\end{tabular}

sont regroupés : leurs caractéristiques communes sont prépondérantes par rapport à leurs différences.

L'analyse de la répartition des espèces montre qu'un petit nombre d'entre elles sont très liées au piège jaune au sol, comme $A$. citricola, et y sont abondantes : Aphis cytisorum Hartig, Aphis rumicis L., Hyperomyzus lactucae L., Phorodon humuli Schranck, Appendiseta robiniae Gillette. Acyrthosiphon euphorbiae Börner, Aulacorthum solani Kaltenbach, Megoura viciae Buckton sont également très liés à ce piège, mais ont des effectifs faibles. Les espèces les plus abondantes liées aux pièges à fils englués sont $A p / O$ neura lentisci Passerini, Cinara spp., Diuraphis noxia Mordvilko, Eulachnus spp., les Pemphiginae, les Phylloxeridae.

Corrélations entre l'échantillon prélevé par le piège à succion et ceux obtenus par les pièges à fils englués ou par les pièges jaunes à eau

La représentation du nuage de points des 93 espèces fournit une vue d'ensemble simple des relations entre les échantillons des pièges. Le piège à succion est considéré ici comme donnant un échantillon objectif des pucerons présents, et les données des autres pièges lui sont comparées.

Les Figures 3,4 et 5 donnent cette représentation pour les pièges à fils englués à $30-55 \mathrm{~cm}$ et le piège à succion, et pour les pièges jaunes au sol et à $70 \mathrm{~cm}$ et le piège à succion. Les coefficients de corrélation correspondants sont respectivement de $0,89,0,70$ et 0,71 .

La dispersion des nuages de points autour des droites de régression et les valeurs des coefficients de corrélation indiquent que l'échantillon obtenu par les pièges à fils englués est plus proche de celui obtenu par le piège à succion que ne le sont ceux provenant des pièges jaunes.

La régression portant sur les captures des pièges jaunes par rapport au piège à succion met en évidence un groupe d'espèces mieux capturées que les autres, déjà mis en évidence par l'analyse factorielle des correspondances : A. citricola, A. cytisorum, A. robiniae, dans les 2 pièges, $A$. rumicis et $H$. lactucae dans le piège jaune au sol. Un groupe d'espèces peu capturées est également mis en évidence : $D$. noxia, Hoplocallis pictus Ferrari, Hyadaphis spp., Hyalopterus pruni Geoffroy, Melanaphis donacis Passerini, Myzus cerasi Fabricius, M. Iythri Schranck, Rhopalosiphum padi L., Tetraneura spp., Uromelan spp. dans les 2 pièges et Anoecia spp., Brachycaudus cardui L., Chaitophorus spp. dans le piège au sol et, dans une moindre mesure, dans le piège à $70 \mathrm{~cm}$. 


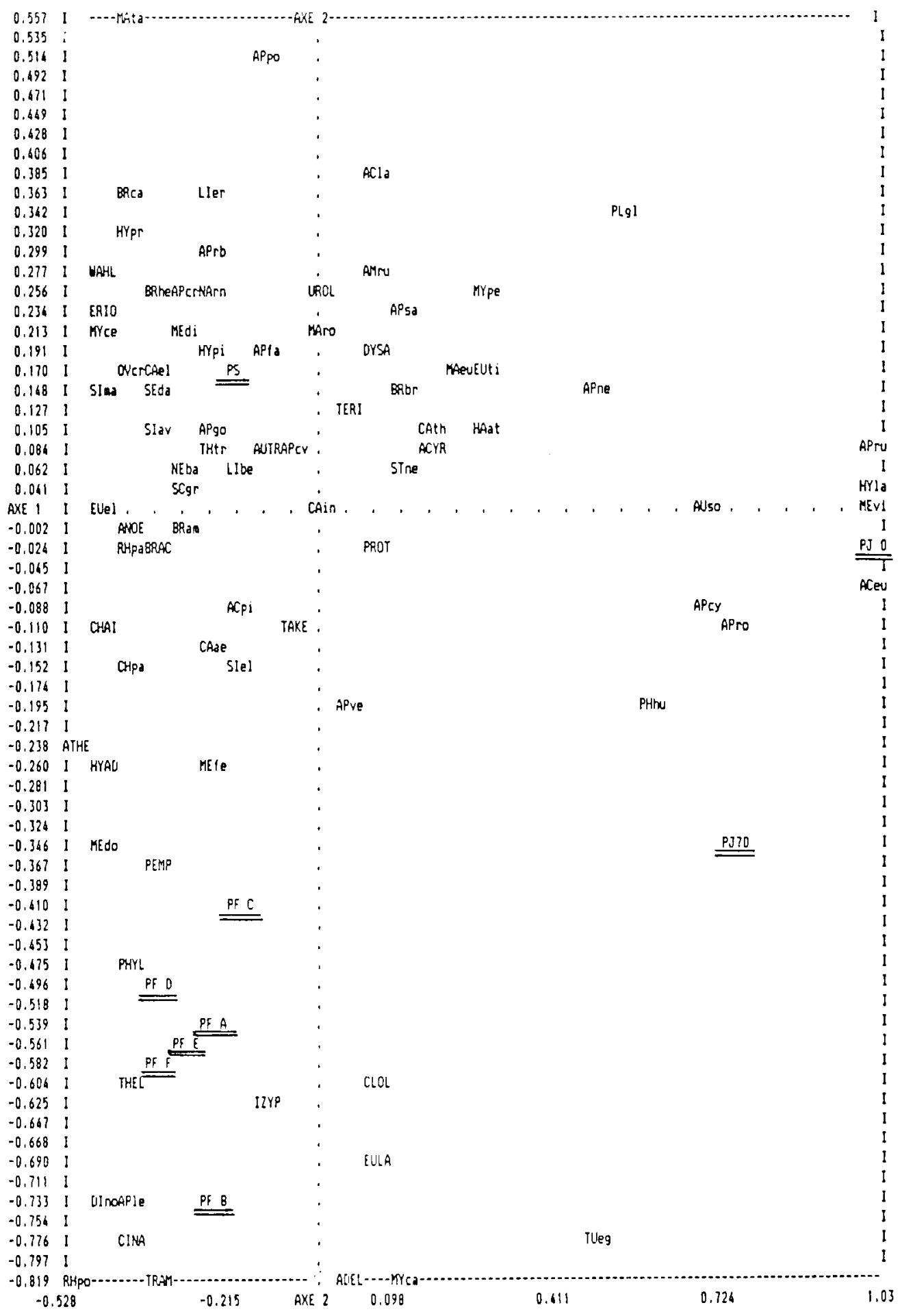

POINTS MULTIFLES ( 100 AN MaXIMM)

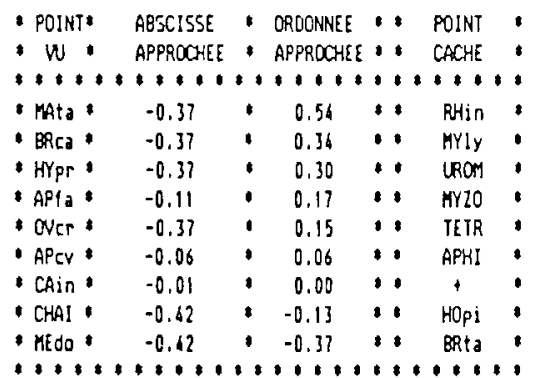

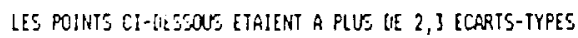
DU CENTRE, ILS ONT ETE RAMENES SLR LE CALAE W GRAPHIQUE.

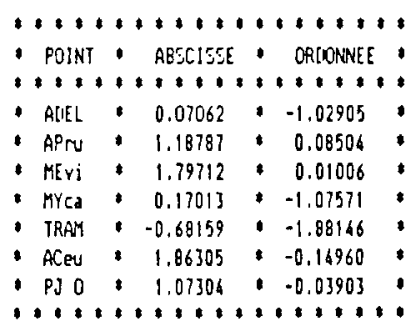

Fig. 2. Représentation graphique des espèces et des pièges dans le plan des axes 1 et 2 de l'analyse factorielle des correspondances. Les données sont celles du Tableau I pour les pièges à fils englués (PF) A à $F$, le piège jaune au sol (PJ), le piège à succion (PS). Chaque espèce est définie par ses initiales. 


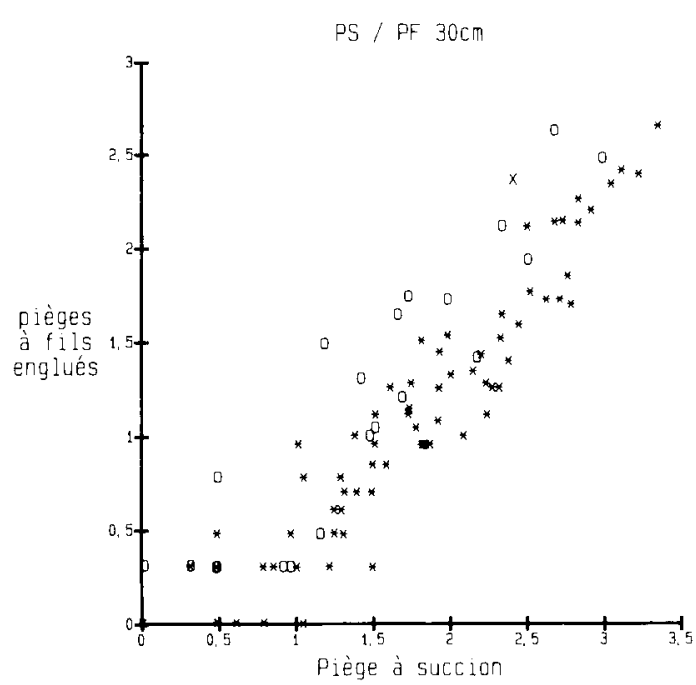

Fig. 3. Relation entre les effectifs de chaque espèce, obtenus par le piège à succion et les 6 pièges à fils englués à $30 \mathrm{~cm}$; données transformées par la fonction Log $(n+1)$. $x$ : Diuraphis noxia; o : espèces n'appartenant pas aux Aphidinae et ayant un hôte ligneux.

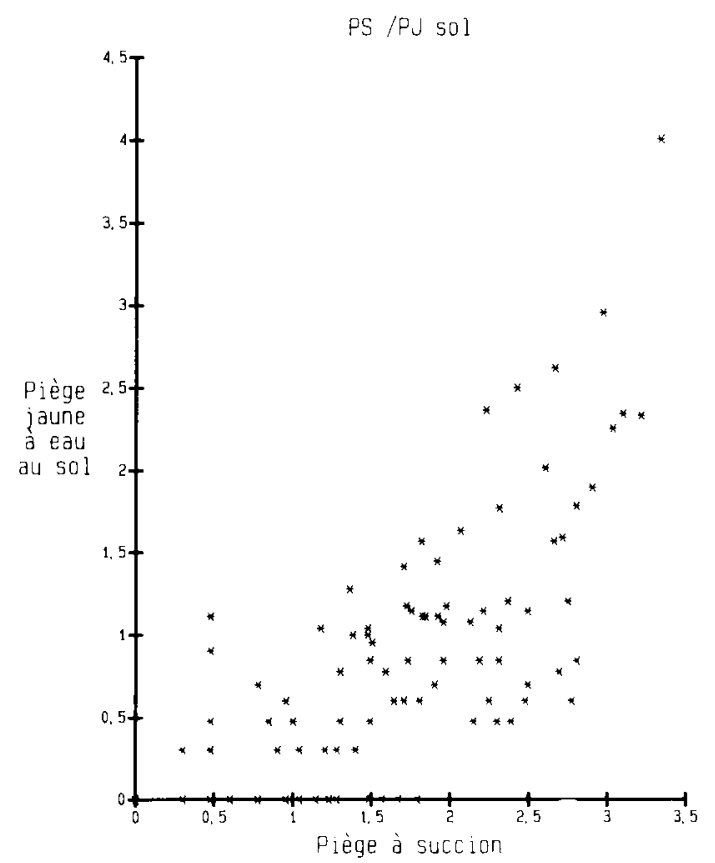

Fig. 4. Relation entre les effectifs de chaque espèce, obtenus par le piège à succion et le piège jaune à eau au sol; données transformées par la fonction $\log (n+1)$.

La comparaison des données des pièges à fils englués au piège à succion suggère une tendance secondaire : les espèces qui admettent comme hôte un arbre et n'appartiennent pas à la sous-famille des Aphidinae (sens de Eastop, 1977) sont généralement mieux capturées par les pièges à fils englués que par le piège à succion (Fig. 3 ), de même que $D$. noxia, qui n'appartient pas au groupe défini ci-dessus.

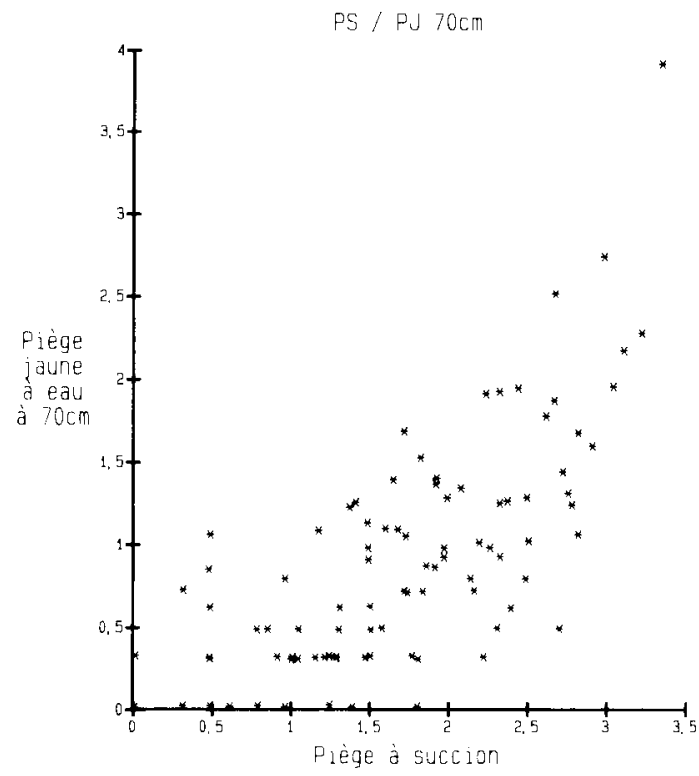

Fig. 5. Relation entre les effectifs de chaque espèce, obtenus par le piège à succion et le piège jaune à eau à $70 \mathrm{~cm}$; données transformées par la fonction $\log (n+1)$.

\section{Comparaison des variations d'effectifs au cours du temps}

Les effectifs des différentes espèces ont varié au cours des 21 jours de prélèvement. On a recherché si les fluctuations observées dans les échantillons des différents pièges étaient synchrones.

Pour les 24 espèces les plus abondantes dans le piège à succion, les corrélations entre les effectifs (transformés en Log $(n+1)$ ) obtenus par ce piège, et respectivement les pièges à fils englués entre 30 et $55 \mathrm{~cm}$ et les 2 pièges jaunes, ont été calculées (Tableau V). Les nuages de points ont été représentés en comparaison pour les pièges à fils englués et le piège jaune au sol (Fig. 6).

Plus l'espèce est représentée par un effectif élevé et meilleure est la corrélation, et ce de la même façon pour les pièges jaunes que pour les pièges à fils englués (Fig. 7). Cette observation tend à montrer que pendant la période d'essai, chaque espèce a toujours eu le même comportement par rapport à chaque type de piège. Les fluctuations sont synchrones, mais plus ou moins amplifiées ou réduites en fonction de l'attractivité du piège sur l'espèce, et la fidélité de réponse dépend du nombre d'individus capturés.

\section{Discussion}

\section{Pièges à fils englués et piège à succion}

Les résultats de cet essai sont les premiers qui permettent de situer les données fournies par des pièges à fils englués par rapport à celles 
Tableau V. Fluctuations quotidiennes des captures de pucerons : coefficients de corrélation entre pièges à fils englués à $30-55 \mathrm{~cm}(\mathrm{PF} 30)$, piège jaune au sol (PJO), piège jaune à $70 \mathrm{~cm}(\mathrm{PJ} 70)$ et piège à succion (PS) pour les 24 espèces les plus abondantes dans le piège à succion (les coefficients de corrélation n'ont pas été calculés lorsque les effectifs sont $<10$ pucerons).

\begin{tabular}{lccc}
\hline Espèce & PF30/PS & $P J O / P S$ & $P J 70 / P S$ \\
\hline Anoecia sp. & 0,70 & - & $-0,24$ \\
Aphis citricola & 0,83 & 0,90 & 0,89 \\
Aphis craccivora & 0,91 & 0,55 & 0,59 \\
Aphis cytisorum & 0,70 & 0,72 & 0,80 \\
Aphis fabae & 0,88 & 0,65 & 0,85 \\
Aphis gossypii & 0,81 & 0,71 & 0,32 \\
Aphis ruborum & 0,62 & 0,65 & 0,73 \\
Aphis sp. & 0,60 & 0,63 & 0,61 \\
Aploneura lentisci & 0,77 & 0,53 & 0,43 \\
Appendiseta robiniae & 0,95 & 0,93 & 0,90 \\
Brachycaudus amygdalinus & 0,80 & 0,76 & 0,52 \\
Brachycaudus cardui & 0,82 & - & 0,63 \\
Brachycaudus helichrysi & 0,65 & 0,50 & 0,31 \\
Brevicoryne brassicae & 0,76 & 0,49 & 0,79 \\
Diuraphis noxia & 0,88 & - & - \\
Dysaphis sp. & 0,61 & 0,69 & 0,79 \\
Hyasaphis sp. & 0,79 & - & - \\
Hyalopterus pruni & 0,81 & - & - \\
Hyperomyzus lactucae & 0,33 &,- 82 & 0,65 \\
Phylloxeridae & 0,89 & - & 0,73 \\
Rhopalosiphum padi & 0,91 & 0,45 & - \\
Semiaphis dauci & 0,54 & - & - \\
Sitobion avenae & 0,54 & 0,68 & 0,44 \\
Therioaphis trifolii & 0,84 & & \\
\hline
\end{tabular}

fournies par un piège à succion, ce dernier étant considéré comme donnant une représentation objective des pucerons ailés présents dans la strate échantillonnée. Les images de l'aphidofaune capturée par les 2 types de pièges sont proches l'une de l'autre (Fig. 3). Des espèces sont cependant relativement plus abondantes dans les pièges à fils englués que dans le piège à succion : espèces autres que les Aphidinae admettant un hôte ligneux et $D$. noxia.

II ne peut pas s'agir d'une hétérogénéité locale de répartition, les sources de ces pucerons étant loin (arbres à plus de $50 \mathrm{~m}$ ) ou uniformément réparties (hôtes secondaires herbacés). L'effet observé peut être dû au gradient altitudinal pour l'espèce $A$. lentisci (Tableau I). Pour les autres espèces, il semble que ce soit les pièges euxmêmes qui soient attractifs. Etant donné la biologie des espèces concernées, l'hypothèse d'une attraction olfactive nous paraît plus probable qu'un phénomène d'origine visuelle : contrairement aux conclusions de Kennedy et al. (1959) étudiant Myzus persicae Sulzer, l'intervention du comportement olfactif a été montrée chez $R$. padi vis-à-vis de son hôte primaire (Petterson, 1970) et chez Nasonovia ribisnigri Mosley vis-àvis des terpènes (Bromley et Anderson, 1982), et les pucerons autres que les Aphidinae n'ont pas été étudiés de ce point de vue.

$D$. noxia est monoécique sur graminées. L'analogie de forme entre les fils englués et les tiges de graminées rendait possible une attractivité du piège spécifique aux pucerons des graminées : les résultats montrent que ce n'est pas le cas, seule cette espèce réagissant ainsi.

Malgré les réserves précédentes, les résultats obtenus montrent que les pièges à fils englués peuvent être assimilés en première approximation à des pièges neutres.

\section{Pièges jaunes et pièges à succion}

Des différences importantes existent entre les représentations de l'aphidofaune fournies par les 2 types de pièges. Elles ne sont pas relatives à une différence des strates échantillonnées : les pièges jaunes aux 2 hauteurs donnent des images comparables, et les gradients altitudinaux décelés dans les pièges à fils englués ne sont pas assez forts pour pouvoir perturber ainsi l'échantillonnage.

Les différences relevées dans cet essai entre ces 2 types de pièges sont comparables à celles constatées lors d'essais réalisés par d'autres 

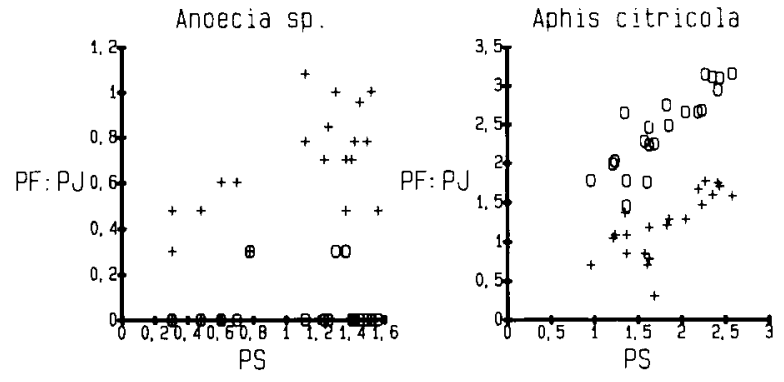

Aph is craccivora
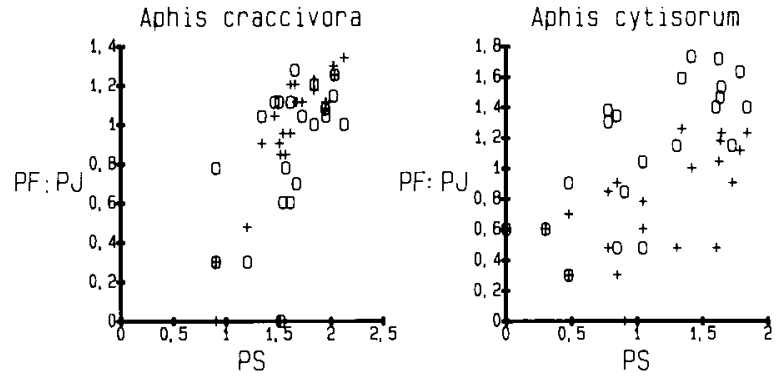

Aph is fabae

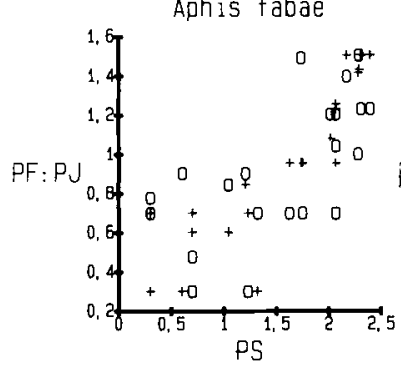

Aphis gossypii
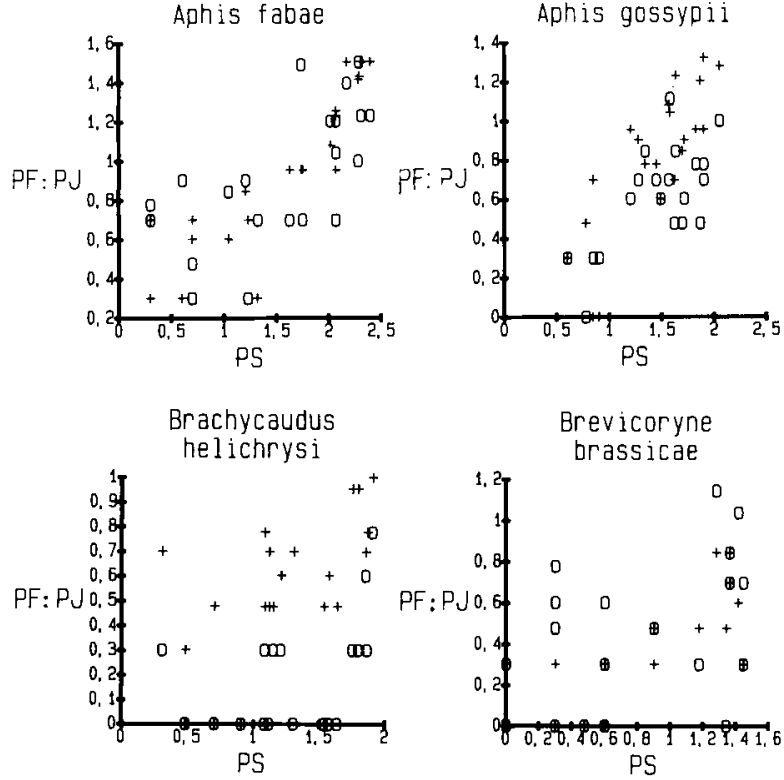

Brevicoryne brassicae

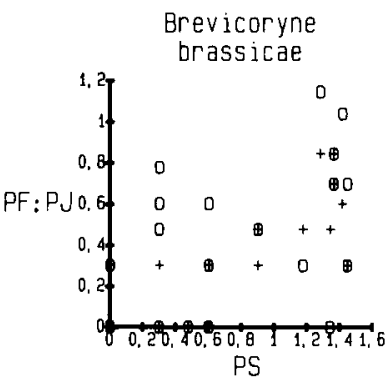

Diuraphis noxia

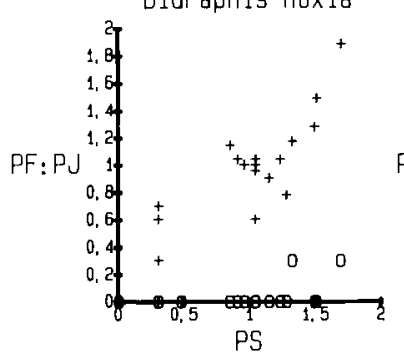

Hyadaphis $\mathrm{sp}$

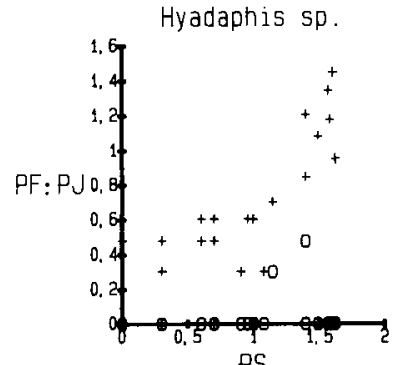

PS
Dysaphis sp

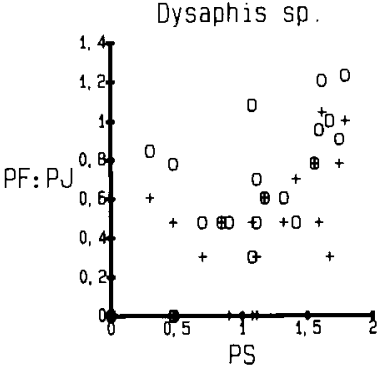

Hyalopterus pruni

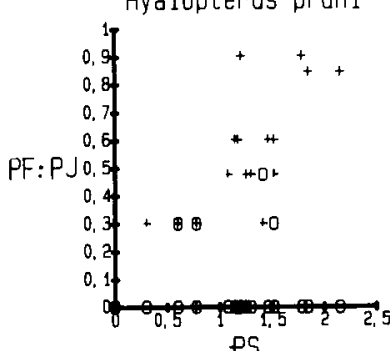

Aphis ruborum

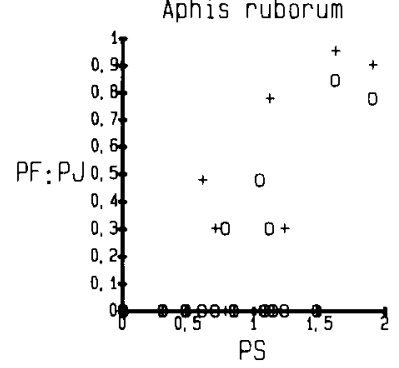

Aploneura lentisci

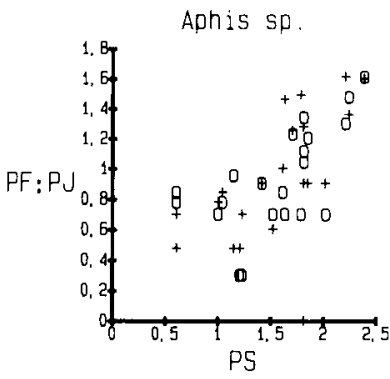

Appendiseta

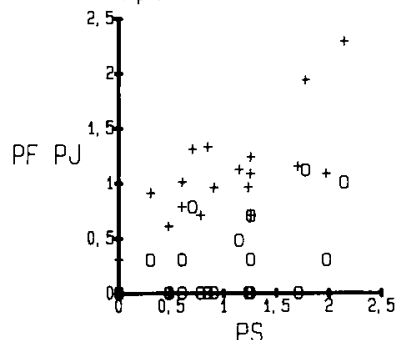

robiniae

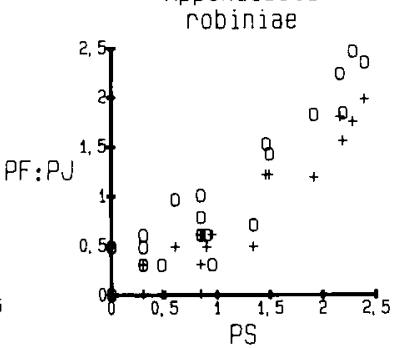

Brachycaudus

Brachycaudus

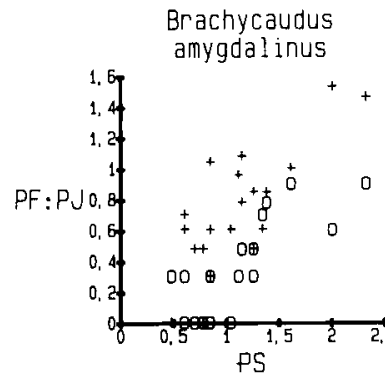

cardui
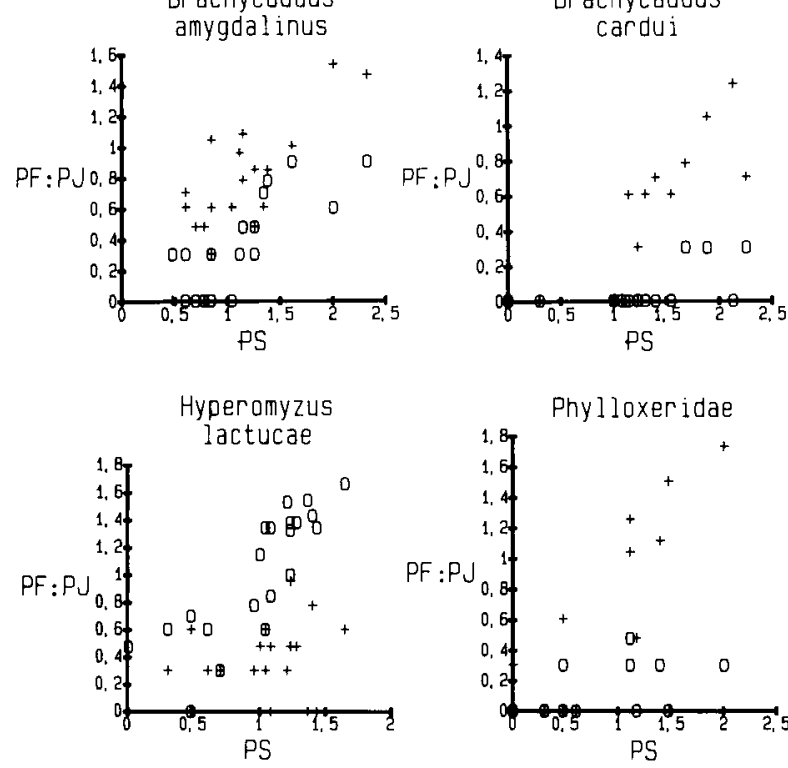

PS

Phylloxer idae

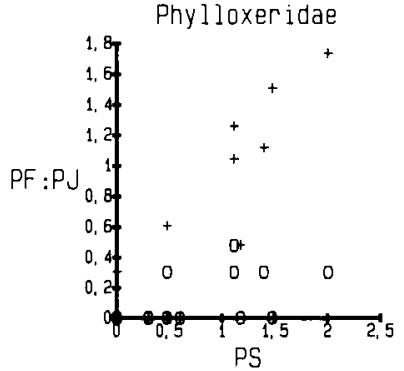

Rhopalosiphum padi

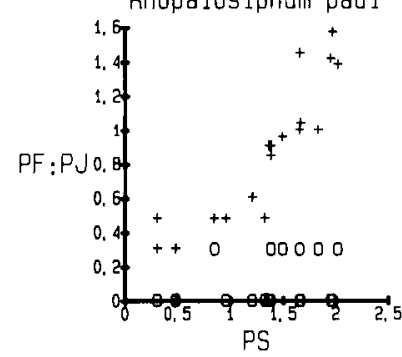

Semiaphis dauci
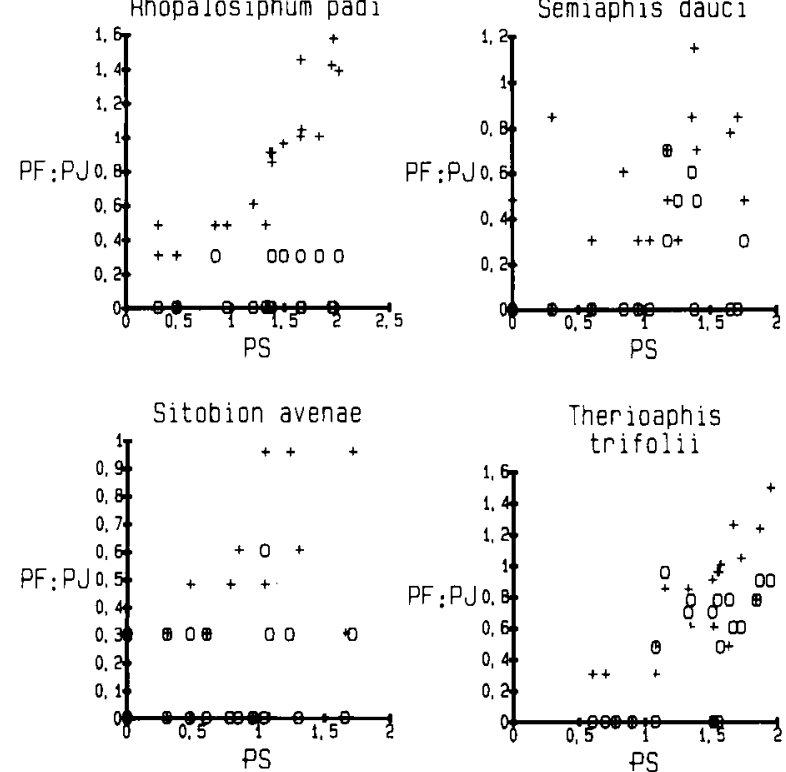

Fig. 6. Relations entre les captures quotidiennes par les pièges à fils englués à $30-55 \mathrm{~cm}$ (PF), ou le piège jaune au sol (PJ) et le piège à succion (PS), pour les 24 espèces les plus abondantes dans le piège à succion. $0:$ piège jaune; + : pièges à fils englués. 


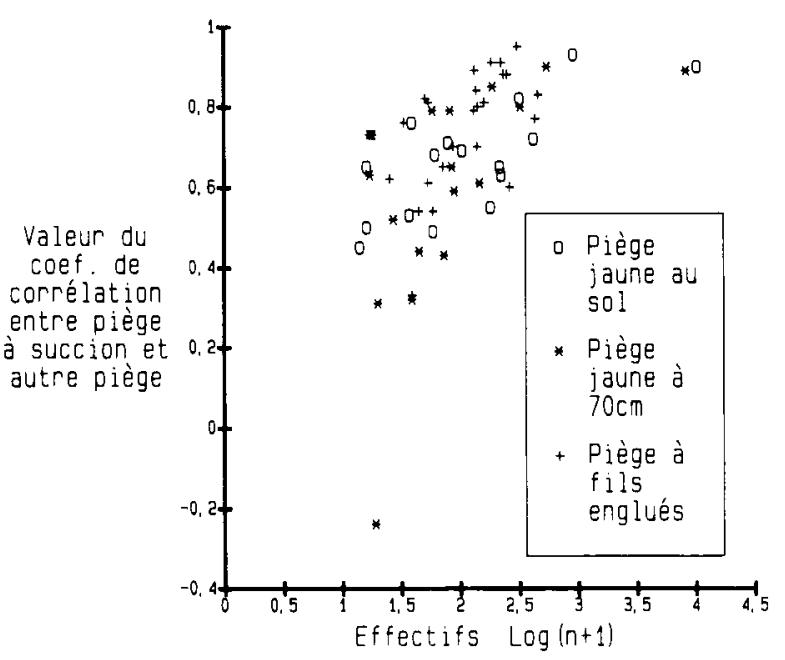

Fig. 7. Comparaison des variations quotidiennes des populations des 24 espèces de pucerons les plus abondantes, estimées par le piège à succion et par le piège jaune au sol, à $70 \mathrm{~cm}$ ou les pièges à fils englués à $30 \mathrm{~cm}$. Ordonnée : coefficient de corrélation entre les effectifs des 24 espèces mesurées par le piège à succion, et le piège jaune au sol, le piège jaune à $70 \mathrm{~cm}$ ou les pièges à fils englués à $30 \mathrm{~cm}$. Abscisse: effectif de l'espèce considérée, dénombré pendant les 21 jours de prélèvement dans les pièges jaunes à eau ou dans les pièges à fils englués.

auteurs dans des conditions différentes (Eastop, 1955; Heathcote, 1957; O'Loughlin, 1963). Lorsque les espèces sont classées sur une échelle relative "très attiré - peut attiré" par le piège jaune (Taylor et Palmer, 1972), on constate que des espèces données se classent, dans l'ensemble, de façon identique les unes par rapport aux autres. Etant donné la diversité des conditions expérimentales, on peut en déduire que globalement le fait d'être attiré par un piège jaune est une caractéristique de l'espèce, plutôt que l'expression d'un comportement d'atterrissage, même si par ailleurs des variations ont été montrées pour une même espèce, suivant la hauteur du piège au-dessus de la végétation ou en fonction de la saison (Robert et al., 1974).

Cette hypothèse est renforcée par le fait que les fluctuations quotidiennes mesurées par les différents pièges sont synchrones. Cela indique que le nombre d'individus capturés par les pièges jaunes est lié au nombre d'individus présents, donc que le comportement d'atterrissage n'est pas pris en compte, ou que la proportion d'individus qui l'exprime dans la population totale n'a pas varié sur la durée de l'essai.

\section{Choix de la technique de piégeage}

Dans le cadre de l'épidémiologie des viroses disséminées par des pucerons, l'utilisation d'un piège pour capturer les vecteurs a pour but de fournir une estimation des contacts entre les vec- teurs potentiels du virus et les plantes sensibles. Deux approches peuvent être retenues pour atteindre ce but : soit intégrer le comportement d'atterrissage sur la plante en fournissant un stimulus adéquat; soit mesurer la circulation des pucerons présents près de la végétation en faisant l'hypothèse qu'ils s'y posent.

L'utilisation de pièges jaunes à eau correspond à la première approche. II est cependant difficile d'établir si le stimulus fourni permet d'atteindre l'objectif fixé. Les remarques du paragraphe précédent permettent d'en douter.

Par ailleurs, le passage d'une représentation de ce type à la représentation donnée par un piège neutre n'est pas possible : les fluctuations des espèces peu capturées bien qu'abondantes ne peuvent pas être obtenues avec précision.

Le piège à succion correspond à la seconde approche. II permet d'obtenir une représentation non biaisée de l'ensemble des espèces qui circulent près des plantes. Mais ses exigences pratiques de fonctionnement en limitent l'utilisation. Les pièges à fils englués constituent une technique de substitution intéressante par ses possibilités d'emploi plus étendues (autonomie, définition plus précise de la strate échantillonnée). Leur principal inconvénient par rapport au piège à succion est la faiblesse des effectifs capturés, mais cet inconvénient peut être évité en augmentant le nombre de pièges utilisés.

\section{Remerciements}

Nous tenons à remercier Colette Roux pour son aide à l'analyse statistique des données et Guy Fauvel pour les fructueuses discussions en commun lors de l'élaboration de cet article.

\section{Références}

Adlerz W.C. (1986) Cucurbit Potyvirus transmission by alate aphids (Homoptera: Aphididae) trapped alive. $J$. Econ. Entomol. 80, 87-92

Bromley A.K. \& Anderson M. (1982) An electrophysiological study of olfaction in the aphid Nasonovia ribisnigri. Entomol. Exp. Appl. 32, 101-110

Brunel E. \& Langouet L. (1970) Influence de caractéristiques optiques du milieu sur les adultes de Psila rosae Fab. (Diptères Psilidés): attractivité de surfaces colorées, rythme journalier d'activité. C. $R$. Soc. Biol. 43, 51-62

Eastop V.F. (1955) Selection of aphid species by different kinds of traps. Nature 176, 936

Eastop V.F. (1977) Worldwide importance of aphids as virus vectors. In: Aphids as Virus Vectors (Harris K.F. \& Maramorosch K., eds). Academic Press, New York, pp. 3-62

Eastop V.F. \& Hille Ris Lambers D. (1976) Survey of the World's Aphids. Dr W. Junk, La Hague 
Halbert S.E., Irwin M.E. \& Goodman R.M. (1981) Alate aphid (Homoptera: Aphididae) species and their relative importance as field vectors of soybean mosaic virus. Ann. Appl. Biol. 97, 1-9

Harrington R., Katis N. \& Gibson R.W. (1986) Field assessment of the relative importance of different aphid species in the transmission of potato virus $\mathrm{Y}$. Potato Res. 29, 67-76

Heathcote G.D. (1957) The comparison of yellow cylindrical, flat and water traps and of Johnson suction traps, for sampling aphids. Ann. Appl. Biol. 45, 133139

Kennedy J.S., Booth C.O. \& Kershaw W.J.S. (1959) Host finding by aphids in the fields. I. Gynoparae of Myzus persicae (Sulzer). Ann. Appl. Biol. 47, 410-423

Knoke J.K., Anderson R.J. \& Louie R. (1977) Virus disease epiphytology: developing field test for disease resistance in maize. In: Proc. Int. Maize Virus Dis, Colloq. and Workshop, (Williams L.E., Gordon D.T., Nault L.R. eds). 16-19 Aug. 1976, Wooster, pp. 116121

Labonne G., Quiot J.B. \& Monestiez P. (1982a) Rôle des diverses espèces de pucerons vecteurs dans la dissémination du virus de la mosaïque du concombre au niveau d'une parcelle de melon dans le sud-est de la France. Agronomie 2, 797-804

Labonne G., Fauvel C., Leclant F. \& Quiot J.B. (1982b) Description d'un piège à succion : son emploi dans la recherche des aphides vecteurs de virus transmis sur le mode non persistant. Agronomie 2, 773-776
Labonne G., Fauvel G., Leclant F. \& Quiot J.B. (1983) Intérêt des pièges à fils dans l'étude des populations de pucerons ailés. Agronomie 3, 315-326

Labonne G. \& Quiot J.B. (1988) Influence de la vitesse et de la direction du vent sur la capture des pucerons ailés par un piège à fils englués. Agronomie 8, 465469

O'Loughlin G.T. (1963) Aphid trapping in Victoria. I. The seasonal occurrence of aphids in three localities and a comparison of two trapping methods. Aust. J. Agric. Res. 14, 61-69

Pettersson J. (1970) Studies on Rhopalosiphum padi (L.). Lantbrukshogsk. Ann. 36, 381-399

Raccah B., Gal-On A. \& Eastop V.F. (1985) The role of flying aphid vectors in the transmission of cucumber mosaic virus and potato virus $Y$ to peppers in Israël. Ann. Appl. Biol. 106, 451-460

Robert Y., Rabasse J.M. \& Rouzé-Jouan J. (1974) Sur l'utilisation des pièges jaunes pour la capture des pucerons en culture de pomme de terre. I. Influence de la hauteur de piégeage. Ann. Zool. Ecol. Anim. 6, 349-372

Robert Y., Dedryver C.A. \& Pierre J.S. (1988) Sampling techniques. In: Aphids. Their Biology, Natural Enemies and Control (Minks A.K. \& Harrewijn P., eds). Elsevier, Amsterdam, pp. 1-20

Taylor L.R. \& Palmer J.M.P. (1972) Aerial sampling. In: Aphid Technology (Van Emden H.F., ed.). Academic Press, London, pp. 189-234 